\title{
Contribution of Sun-like faculae to the light-curve modulation of young active dwarfs ${ }^{\star}$
}

\author{
P. Gondoin \\ European Space Agency, ESTEC - Postbus 299, 2200 AG Noordwijk, The Netherlands \\ e-mail: pgondoin@rssd.esa.int \\ Received 9 July 2007 / Accepted 5 November 2007 \\ ABSTRACT

\begin{abstract}
Aims. The time variability of the broadband solar irradiance depends not only on the intrinsic evolution and visibility modulation of sunspots but also on that of faculae that become brighter near the limb during the solar rotation. Sun-like faculae around spots could also play a significant role in modulating the broadband visible flux of active dwarfs. It is the aim of the present study to test this hypothesis.

Methods. I analyzed high accuracy light-curves of two active dwarfs obtained with the MOST satellite during several stellar rotation periods. The observed time series were fitted using a model that takes into account not only starspot contributions but also the areas of faculae in active regions and their bolometric contrast.

Results. A low value of the mean ratio of faculae to cool spot areas in active regions provides the best description of $\epsilon$ Eri and $\kappa$ Ceti light curves.

Conclusions. Although not conclusive, this result suggests that the ratio of faculae to cool spot areas decreases in stars somewhat more active than the Sun.
\end{abstract}

Key words. stars: activity - stars: late-type - stars: starspots - techniques: photometric - methods: numerical

\section{Introduction}

Starspots cooler than the unperturbed photosphere can account for the rotation modulated attenuation of stellar fluxes in broad optical pass-bands but recent methods for simulating light curves in broad optical pass-bands assume stellar active regions that consist of both sunspots and faculae (Aigrain et al. 2004; Moutou et al. 2005). Indeed, on the Sun, bright faculae produce an increase of the disk-integrated flux. The time variability of the broadband solar irradiance thus depends not only on the intrinsic evolution and visibility modulation of sunspots but also on that of faculae that become brighter near the limb during the solar rotation. The ratio of facular to sunspot area seems to be the main factor determining the sign and magnitude of variation in total solar irradiance, at least on time-scales extending up to the length of the $11 \mathrm{yr}$ activity cycle (Foukal \& Lean 1986; Chapman 1987; Foukal 1992). As an example, the mean ratio of facular to sunspot area was found to be $16.7 \pm 0.5$ for a 7.5 year period during solar cycle 22 but showed a significant increase as the solar cycle progressed (Chapman et al. 1997).

Studies of variability among Sun-like stars (Radick et al. 1998; Lockwood et al. 2007) show that young, active stars become fainter as their chromospheric emission increases, whereas the older stars, including the Sun, tend to become brighter as their chromospheric emission increases. An interpretation of this behaviour is that the long-term variability of young stars is spot dominated, whereas for older stars like the Sun, it is faculaedominated. Solar faculae provide a strong flux contribution to

^ Based on data from the MOST satellite, a Canadian Space Agency mission, jointly operated by Dynacon Inc., the University of Toronto Institute for Aerospace Studies and the University of British Columbia, with the assistance of the University of Vienna. the emission cores of the $\mathrm{Ca}$ II $\mathrm{H}$ and $\mathrm{K}$ lines. A rotational modulation has been observed in the $\mathrm{Ca}$ II $\mathrm{H}$ and $\mathrm{K}$ emission of many active dwarfs (Vaughan et al. 1981; Baliunas et al. 1983) that shows a period in agreement with photometric measurements in broad optical pass-bands. This suggests that Sun-like faculae around spots could also play a significant role in modulating the broadband visible flux of these stars.

It is the aim of the present study to test this hypothesis by analyzing the flux variations in broad optical pass-bands of active solar-type stars. For that purpose, I analyzed the high accuracy light curves of two young active dwarfs, $\epsilon$ Eri and $\kappa$ Ceti, that were obtained with the MOST satellite during long uninterrupted observing runs of about one month. The study uses a model (Lanza et al. 2006) based on the rotational modulation of the visibility of active regions that consist of faculae and sunspots with brightness contrasts that are functions of the limb angle. Brief descriptions of the datasets and of the model are given in Sects. 2 and 3. The results are presented in Sect. 4.

\section{The targets and datasets}

The rotation modulation of stellar light curves by large starspots on active dwarfs induce broadband flux variations of typically a few percent. The contributions of Sun-like faculae to these photometric variations could be of the order of a few thousandth or even less since, on young active dwarfs, faculae are expected to have a lower influence on stellar luminosity than spots (Radick et al. 1998). Their detections thus require the measurement of stellar light curves with a high relative accuracy over several rotation periods. The MOST satellite registered the visible light curve of two active solar-type stars, $\epsilon$ Eri and $\kappa$ Ceti, in 2005 and 2003, respectively for durations exceeding three times their 
rotation periods and with point to point accuracies of $50 \mathrm{ppm}$ and 200 ppm, respectively (Rucinsky 2004). The Canadian microsatellite MOST (Walker et al. 2003) was launched on 30 June 2003. Its payload consists of a 15-cm Rumak-Maksutov telescope feeding two CCD detectors (one for stellar photometry, the other for guiding) through a single $350 \mathrm{~nm}$ to $700 \mathrm{~nm}$ broadband filter. MOST provides rapid photometry of bright stars $(V<6.5)$ located in a 54 degree wide band of the sky with up to 2 months time coverage.

The visible light curve of $\epsilon$ Eri ( $V=3.72$; HD 22049; HIP 16537; HR 1084) was observed from 2005 Oct. 28 till Dec. 3 , i.e. for a total of 35.7 days. $\epsilon$ Eridani is a K2 V dwarf that exhibits a high level of magnetic activity (Gray \& Baliunas 1995) consistent with a relatively young age of less than $1 \mathrm{Gyr}$ (Soderblom \& Dappen 1989). It is the closest star (3.3 pc) to the Sun known to have a planetary companion (Hatzes et al. 2000). The inclination of the planetary companion orbit is $26.2^{\circ}$ (Benedict et al. 2006). A 130 AU diameter dust ring also surrounds the system (Greaves et al. 2005). Vaughan et al. (1981) found a period of $11.8 \mathrm{~d}$ in the Ca II K line reversal of $\epsilon$ Eridani. Several observers have found a periodic variability in its photometric light curve attributed to rotation modulation by starspots. Photometric variability in the $V$ bandpass may have first been detected by Fisher et al. (1983) and Baliunas et al. (1983) reported a period of $11.3 \mathrm{~d}$. Frey et al. (1991) found five single spots visible for 1 to 2 months that yielded rotation periods in the range $10.0 \mathrm{~d}<P<12.3 \mathrm{~d}$. Recently, Croll et al. (2006) analyzed $\epsilon$ Eri MOST time series and found two starspots with rotation periods of $11.35 \mathrm{~d}$ and $11.55 \mathrm{~d}$, respectively, from which they derived a differential rotation coefficient.

A long uninterrupted MOST observation of another active dwarf, $\kappa$ Ceti ( $V=4$ : 83; HD 20630; HIP 15457; HR 996), was conducted in 2003, starting on Nov. 3 for a duration of $30.44 \mathrm{~d}$ (Walker et al. 2007). $\kappa$ Cet is a nearby (9.16 pc) single G5 dwarf. Evidence for rotation modulation of its $\mathrm{Ca}$ II $\mathrm{H}$ and $\mathrm{K}$ chromospheric emission was found by Vaughan et al. (1981) who reported a period of $8.5 \mathrm{~d}$. The broadband variability of $\kappa$ Ceti was detected by the Hipparcos satellite (ESA 1997) with a period of $9.09 \mathrm{~d}$. Since then, several studies have aimed at reconciling apparent changes in its photometric period. Gaidos et al. (2000) derived rotation periods of $\kappa$ Ceti ranging between $9.14 \mathrm{~d}$ and $9.46 \mathrm{~d}$, each with uncertainty at the level of $0.03-0.05 \mathrm{~d}$. Messina \& Guinan (2003) conducted a careful study over six seasons and determined rotation periods ranging between $9.045 \mathrm{~d}$ and 9.406 d. Using MOST observations, Rucinski et al. (2004) found two starspots with rotation periods of $8.9 \mathrm{~d}$ and $9.3 \mathrm{~d}$, respectively. Analysis results of the same time series by Walker et al. (2007) gave similar results with, however, a slightly shorter period of the second spot. Changes of the $\kappa$ Ceti photometric period over time suggest a combination of differential rotation and concentration of starspots at different stellar latitudes from year to year (Gaidos et al. 2000), consistent with a latitude drift of starspots during an activity cycle. Messina \& Guinan (2002) found the existence of a Sun-like starspot cycle of $5.9 \mathrm{yr}$, which is similar to the chromospheric activity cycle of $5.6 \mathrm{yr}$ found by Baliunas et al. (1995). Güdel et al. (1997) estimated an age of $750 \mathrm{Myr}$ for $\kappa$ Ceti from the relatively rapid rotation period of $9.2 \mathrm{~d}$ seen in the spot modulation.

The reductions of the $\epsilon$ Eri and $\kappa$ Ceti light-curves followed the scheme outlined by Rucinski et al. (2004). More than 100000 data points were collected with exposure times of $20 \mathrm{~s}$ sampled every $25 \mathrm{~s}$. For the starspots analysis, the data were binned at the MOST orbital period of $101.413 \mathrm{~min}$
Table 1. Stellar parameters of $\epsilon$ Eri and $\kappa$ Ceti.

\begin{tabular}{cc|ccccc}
\hline \hline & & $\begin{array}{c}T_{\text {eff }} \\
(\mathrm{K})\end{array}$ & $\begin{array}{c}R \\
\left(R_{\odot}\right)\end{array}$ & $\begin{array}{c}\log g \\
(\mathrm{cgs})\end{array}$ & $\begin{array}{c}i \\
\left({ }^{\circ}\right)\end{array}$ & $\begin{array}{c}\text { Age } \\
(\mathrm{Gyr})\end{array}$ \\
\hline$\epsilon$ Eri & $(\mathrm{K} 2 \mathrm{~V})$ & 5030 & 0.76 & 4.0 & 30 & $<1.0$ \\
$\kappa$ Ceti & $(\mathrm{G} 5 \mathrm{~V})$ & 5750 & 0.95 & 4.5 & 60 & $\approx 0.75$ \\
\hline
\end{tabular}

(Croll et al. 2006). The reduced light curves were down-loaded from the MOST Public Archive.

\section{The stellar variability model}

The rotation modulation of stellar photometric light-curves by stellar active regions can be modeled using two numerical approaches: the surface integration methods and the analytical method (Ribarik et al. 2003). The former assigns a temperature to each pixel of the spherical integration net and then varies each value until an optimal fit to the data is achieved. In the present study, I used the analytical approach described by Lanza et al. (2006) and based on a model used to fit the time variations of the solar bolometric and spectral irradiance. Following this approach, the variation of the monochromatic stellar flux due to discrete active regions is given by:

$\Delta F(\lambda, t)=\Sigma_{k} \mu_{k} A_{k} I\left(\lambda, \mu_{k}\right)\left[c_{\mathrm{s}}(\lambda)+Q c_{\mathrm{fac}}\left(\lambda, \mu_{k}\right)\right]$

where $\Delta F(\lambda, t)$ is the perturbed stellar flux at wavelength $\lambda$ and time $t, \mu_{k}=\cos \psi_{k}$, with $\psi_{k}$ the angle between the normal to the $k$ th active region and the line of sight, $A_{k}$ the area of the cool spots in the $k$ th active region, $I\left(\lambda, \mu_{k}\right)$ the specific intensity of the unperturbed photosphere (which depends on $\mu$ owing to limb-darkening), $c_{\mathrm{s}}$ the contrast of the cool spots (see Eq. (4) in Sect. 4), assumed to be independent of the limb angle, $c_{\mathrm{fac}}$ the facular contrast (see Eq. (5) in Sect. 4), and $Q$ the mean ratio of facular to cool spot areas in active regions. Spots and faculae are assumed to be spatially coincident. The summation is extended over the active regions on the visible hemisphere, i.e., for which $\mu_{k}>0$. The value of $\mu_{k}$ is a function of time that is given by:

$\mu_{k}=\cos i \sin \theta_{k}+\sin i \cos \theta_{k} \cos \left(\Omega_{k} t+\Lambda_{k}\right)$

where $i$ is the inclination of the stellar rotation axis along the line of sight, $\theta_{k}$ is the latitude, $\Lambda_{k}$ the longitude, and $\Omega_{k}=2 \pi / P_{k}$ the angular velocity of the $k$ th active region having a rotation period $P_{k}$. The specific intensity of the undisturbed photosphere is given by:

$I\left(\lambda, \mu_{k}\right)=\frac{4\left(a_{\mathrm{p}}+b_{\mathrm{p}} \mu+c_{\mathrm{p}} \mu^{2}\right)}{a_{\mathrm{p}}+2 b_{\mathrm{p}} / 3+c_{\mathrm{p}} / 2} B\left(\lambda, T_{\mathrm{u}}\right)$

where the $a_{\mathrm{p}}, b_{\mathrm{p}}$, and $c_{\mathrm{p}}$ are the quadratic limb darkening coefficients. Stellar oscillations are not taken into account in the light-curve simulations that focus on a low frequency domain of stellar variability. The effects of super-granulation, mesogranulation and granulation are also neglected.

\section{Light-curve analysis}

The analyses of $\epsilon$ Eri and $\kappa$ Ceti light-curves were conducted using the stellar parameters given in Table 1. $\epsilon$ Eri effective temperature and inclination angle are from Foing et al. (1989) and Fischer \& Valenti (2005), respectively. The effective temperature, radius, surface gravity and inclination angle of $\kappa$ Ceti are from Biezzo et al. (2007), Landi et al. (1997), 
Table 2. Parameters of two spot models that best fit the $\epsilon$ Eri light curve obtained in 2005. Two models are considered. Model A has a fixed facular to spot area ratio $Q=5$. Model B has a fixed facular to spot area ratio $Q=1$. Errors are given at a $90 \%$ confidence level.

\begin{tabular}{lcccc}
\hline \hline$\epsilon$ Eri (2005) & $\begin{array}{c}\text { Model A } \\
\text { spot 1 }\end{array}$ & spot 2 & $\begin{array}{c}\text { Model B } \\
\text { spot 1 }\end{array}$ & spot 2 \\
\hline$Q$ (fixed) & 5.0 & 5.0 & 1.0 & 1.0 \\
Surface $(\%)$ & $1.73 \pm 0.07$ & $0.95 \pm 0.05$ & $2.26 \pm 0.08$ & $1.09 \pm 0.06$ \\
Latitude $\left({ }^{\circ}\right)$ & $68.7 \pm 1.1$ & $55.2 \pm 4.2$ & $67.6 \pm 0.9$ & $40.6 \pm 7.4$ \\
Longitude $\left(^{\circ}\right)$ & $346.4 \pm 2.1$ & $198.5 \pm 2.3$ & $346.5 \pm 1.8$ & $192.5 \pm 1.9$ \\
Period (d) & $11.57 \pm 0.04$ & $11.40 \pm 0.04$ & $11.55 \pm 0.03$ & $11.39 \pm 0.03$ \\
\hline$\chi^{2}$ (d.o.f.) & $356(484)$ & & $350(484)$ & \\
\hline
\end{tabular}

Table 3. Parameters of two spot models that best fit the $\kappa$ Ceti light curve obtained in 2003. Two models are considered. Model A has a fixed facular to spot area ratio $Q=5$. Model B has a fixed facular to spot area ratio $Q=0$. Errors are given at a $90 \%$ confidence level.

\begin{tabular}{lcccc}
\hline \hline$\kappa$ Ceti (2003) & $\begin{array}{c}\text { Model A } \\
\text { spot 1 }\end{array}$ & spot 2 & $\begin{array}{c}\text { Model B } \\
\text { spot 1 }\end{array}$ & spot 2 \\
\hline$Q$ (fixed) & 5.0 & 5.0 & 0.0 & 0.0 \\
Surface $(\%)$ & $3.6 \pm 0.2$ & $13.2 \pm 2.3$ & $2.8 \pm 0.2$ & $9.1 \pm 1.5$ \\
Latitude $\left({ }^{\circ}\right)$ & $54.2 \pm 2.7$ & $87.3 \pm 0.5$ & $28.2 \pm 8.8$ & $86.4 \pm 0.6$ \\
Longitude $\left(^{\circ}\right)$ & $68.9 \pm 3.2$ & $308.8 \pm 7.8$ & $69.2 \pm 2.8$ & $287.7 \pm 5.8$ \\
Period (d) & $9.05 \pm 0.05$ & $9.73 \pm 0.17$ & $9.05 \pm 0.04$ & $9.50 \pm 0.13$ \\
\hline$\chi^{2}$ (d.o.f.) & $242(408)$ & \multicolumn{3}{c}{$205(408)$} \\
\hline
\end{tabular}

Bean et al. (2006) and Gaidos et al. (2000), respectively. The coefficients of the quadratic limb darkening law adopted to describe the unperturbed bolometric specific intensity of $\epsilon$ Eri $\left(T_{\mathrm{eff}} \approx 5000 \mathrm{~K}, \log g \approx 4.0\right)$ and $\kappa$ Ceti $\left(T_{\mathrm{eff}} \approx 5750 \mathrm{~K}, \log g \approx\right.$ 4.5) were derived from Claret \& Gimenez (1990).

Active region parameters include the spot effective temperature, and the spectral facular contrast model. The contrasts of the cool spots are assumed to be independent of their position on the stellar disk and are estimated as:

$c_{\mathrm{s}}(\lambda)=\frac{B\left(\lambda, T_{\mathrm{s}}\right)}{B\left(\lambda, T_{\mathrm{eff}}\right)}-1$

where $B(\lambda, T)$ is the Planck function, $T_{\mathrm{s}}$ the spot effective temperature and $T_{\text {eff }}$ is the effective temperature of the unperturbed photosphere. The temperatures of starspots estimated using the Doppler imaging technique (e.g. Strassmeier et al. 2003) or from the variation of color indexes vs rotation (e.g. Eaton 1992) are between 600 and $1600 \mathrm{~K}$ cooler than the unperturbed photosphere for most active stars. For the simulation, I assumed $T_{\mathrm{s}}=T_{\text {eff }}-800 \mathrm{~K}$. Following Lanza et al. (2004), the facular contrast is described by a linear function of $\mu$, being zero at the disk center $(\mu=1)$ and maximum at the $\operatorname{limb}(\mu=0)$, i.e.:

$c_{f a c}(\lambda)=(1-\mu)\left(\frac{c_{\mathrm{o}} \Delta \lambda}{1.05}\right)\left(\frac{\Delta T_{\mathrm{f}}}{\Delta T_{\mathrm{f} \odot}}\right)\left(\frac{T_{\odot}}{T_{\mathrm{eff}}}\right)^{2} \lambda^{-1}$

where $\Delta \lambda=650 \mathrm{~nm}$ is the wavelength range between 350 and $1000 \mathrm{~nm}$ where the average facular contrast is assumed to be equal to its bolometric value $c_{\mathrm{o}}=0.115$, according to Foukal et al. (1991). Equation (5) has been derived for the Sun, but rescaled to other stars by using their effective temperature and facular excess temperature. $\Delta T_{\mathrm{f}}$ is the temperature excess between the faculae and the stellar photosphere, $\Delta T_{\mathrm{f} \odot}$ is the facular temperature excess in the Sun, and $T_{\odot}=5778 \mathrm{~K}$ is the effective temperature of the Sun. In the simulations, I assumed $\Delta T_{\mathrm{f}}$ $=\Delta T_{\mathrm{f} \odot}$. The wavelength dependence of the facular contrast in Eq. (5) is in agreement with the observations of the monochromatic contrasts of solar faculae, as discussed by Chapman \& McGuire (1977).
Best fit models to $\epsilon$ Eri and $\kappa$ Ceti light-curves were obtained by minimizing the sum of squared residuals. The fixed parameters in the simulations included the stellar and active region parameters previously described. Because of the present ignorance of the structure of stellar active region, the facular to cool spot area ratio was initially set to $Q=5.0$. The surfaces, longitudes, latitudes and rotation periods of the active regions were left as free parameters. The analyses of the light-curves were performed as a grid search using a two spots model and iterating on 8 variable parameters. In order to avoid local minima in the sum of squared residuals, sequences of numerous fitting processes were conducted with randomly generated initial conditions for the latitudes, longitudes and surface areas of the active regions. The best fit solutions, i.e. the one with the lowest sum of squared residuals to the measured light curves, are described in Tables 2 and 3 (model A) for $\epsilon$ Eri and $\kappa$ Ceti, respectively. The surface coverages given in Tables 2 and 3 are the spot surfaces relative to the stellar surface. Longitude $0^{\circ}$ is defined as the longitude of the meridian that intersected the observer's line of sight on the visible hemisphere at the beginning of the observation.

In order to assess whether Sun-like faculae around spots could also play a significant role in modulating the broadband visible flux of $\epsilon$ Eri and $\kappa$ Ceti, I conducted a series of fitting processes starting from the best fit model found for $Q=5$ but varying the facular to cool spot area ratio $Q$. For $\epsilon$ Eri, the $\chi^{2}$ of the best fit solutions increases for values of $Q$ greater than 5 and decreases for value of $Q$ lower than 5. A minimum $\chi^{2}$ was found around $Q=1$. Although the least square method suggests that a low value of $Q$ provides an improved description of $\epsilon$ Eri light-curve using a two spot model, the improvement between the $Q=10$ and $Q=1$ model is not significant at a high confidence level according to the F statistics. The upper limit for which the difference in chi-squared becomes significant at a more than $80 \%$ confidence level is $Q=53$. Regarding $\kappa$ Ceti light-curve, a minimum $\chi^{2}$ was found at $Q=0$. The improvement between the $Q=5$ and $Q=0$ models is significant at a $95 \%$ confidence level. The $\chi^{2}$ of the best fit solutions also reach a local minimum for a value $Q \approx 13$. This solution implies an unreasonably large shear since, according to the spot model, the spots are only 12 to 15 degrees apart in latitude, but rotate with 

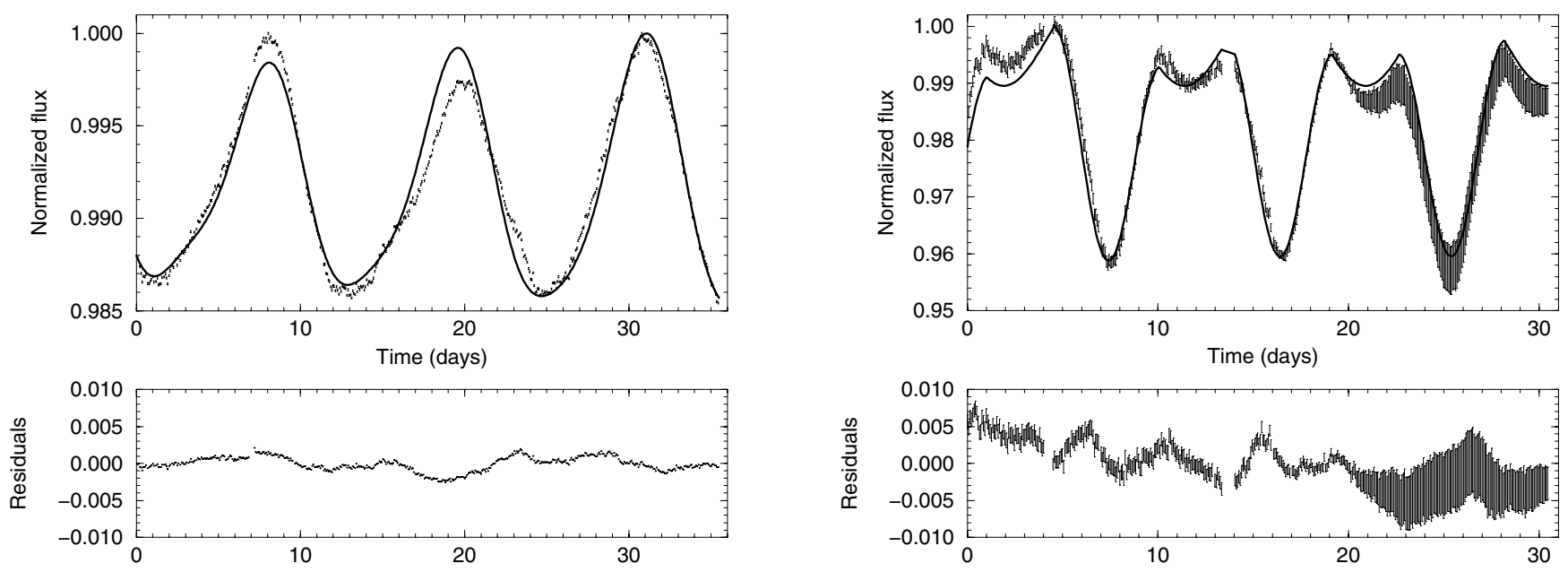

Fig. 1. Left: best fit model to $\epsilon$ Eri light-curve using a two spots model with a facular to spot area ratio of 1 . Right: best fit model to $\kappa$ Ceti 2003 light-curve using a two spots model with a facular to spot area ratio of 0 . The residuals to the best fit models are given in the lower panels.

significantly different periods. A low value of $Q$ thus provides a more likely description of the $\kappa$ Ceti light-curve using a two spot model, although there is no improvement with respect to the $Q=13$ best fit solution according to the F statistics. The upper limit for which the difference in chi-squared becomes significant at more than $95 \%$ confidence level is $Q=20$. However, for large values of $Q$, the spot area and the faculae area become so large on $\kappa$ Ceti that the model approximation used to describe the active regions is no longer valid.

Except for the highest time-resolution lightcurves, or those showing eclipses, the lightcurves of active stars can generally be modeled with a two-spot model. Spectroscopic techniques (e.g. Doppler imaging), when used simultaneously, show more complex spot patterns (e.g. Marsden et al. 2005; Unruh et al 1995; Weber \& Strassmeier 1998). I fitted the lightcurve of $\epsilon$ Eri with a three spot model to quantify the change in chi-squared due to extra free spot parameters compared to the value obtained when optimizing the faculae to spot area ratio in a two spot model. In order to limit the number of free parameters, the faculae to spot area ratio was fixed to $Q=5$ and the position and rotation of two of the three spots were fixed to the value obtained with the corresponding $(Q=5)$ two spot model. Their surface areas were left as free parameters. A best fit solution with three spots was found with no improvement in chi-squared compared to the two-spot model with the optimum faculae to spot area ratio. A similar test applied to $\kappa$ Ceti light-curve gives the same result.

\section{Summary}

The rotational modulation of stellar light-curves by large starspots on active dwarfs induces broadband flux variations of typically a few percent. If present, contributions of Sun-like faculae to these photometric variations are expected to be at most a few milli-magnitudes and could be detected by in-orbit photometric experiments due to their ability to measure visible lightcurves with a high accuracy during long uninterrupted periods. I analyzed the MOST light-curves of two young active dwarfs, $\epsilon$ Eri and $\kappa$ Ceti, using a model that takes into account not only the contributions of sunspots but also the areas of faculae in active regions and their bolometric contrast. In order to assess whether Sun-like faculae around spots could play a role in modulating the broadband visible flux of $\epsilon$ Eri and $\kappa$ Ceti,
I conducted a series of fitting processes varying the facular to cool spot area ratio $Q$. The best solutions, i.e. the one with the lowest sum of squared residuals to the measured light curves, are obtained when assuming low values of the facular to cool spot area ratio. This suggests that the ratio of faculae to cool spot areas is small in young active dwarfs somewhat more active than the Sun. However, although the least square method suggests that a low value of $Q$ provides an improved description of the $\epsilon$ Eri and $\kappa$ Ceti light-curves using a two spot model, the improvement with respect to other best fit solutions with higher values of $Q$ is not significant according to the F statistics.

One explanation for the absence of conclusive results regarding the existence of Sun-like faculae on active dwarfs may be that the model used here contains to many simplifying assumptions. In particular, in the absence of color information, the facular-to-spot ratio had to be determined independently of the spot temperature. Also, the facular contrast dependence as a function of limb angle is uncertain, and a simple temperature excess was assumed between the faculae and the photosphere. Some parameters are expected to be interdependent. This simple model was selected not only because it minimizes the number of free parameters and increases the confidence in the fit but also because of a lack of adequate knowledge for a more detailed description of active regions. For example, the radiation deficit due to spots and the radiation excess due to faculae is assumed to depend solely on their area and location on the star surface. This is not the case for solar faculae. For stars as for the Sun, one additional parameter, i.e. the local magnetic field strength, is most likely involved.

Another simplifying assumption is neglecting the possible evolution of spots and faculae. This simplification could lead to errors in interpreting long rotation modulated light-curves. In the present analysis, appreciable mismatches occur between the $\epsilon$ Eri and $\kappa$ Ceti MOST light curves and the best fit 2 spot models (see Fig. 1). In the absence of long term drift of the MOST instrument response, such intrinsic variations could be the result of significant developments in active regions that occurred during the observations. In order to estimate these effects, the light curve of $\epsilon$ Eri was split into three datasets of about 12 days duration each (see Fig. 1 left) that were fitted independently. The spot location and size were allowed to vary but the faculae to spot area ratio and the rotation period of the active regions were 
Table 4. Parameters of two spot model that best fit the $\epsilon$ Eri light curve obtained in 2005. The light curve of $\epsilon$ Eri was split in three consecutive datasets of about 12 days duration each, i.e. one rotation period (see Fig. 1 left), that were fitted independently. Errors are given at a $90 \%$ confidence level. $\chi^{2}$ values are given for the best fit solutions to each individual dataset and, on the last line, when comparing these solutions with the overall light curve of $\epsilon$ Eri.

\begin{tabular}{l|cc|cc|cc}
\hline \hline$\epsilon$ Eri (2005) & First period & & Second period & & Third period & \\
& spot 1 & spot 2 & spot 1 & spot 2 & spot 1 & spot 2 \\
\hline$Q$ (fixed) & 1.0 & 1.0 & 1.0 & 1.0 & 1.0 & 1.0 \\
Surface (\%) & $2.46 \pm 0.03$ & $1.14 \pm 0.03$ & $1.29 \pm 0.03$ & $0.73 \pm 0.06$ & $1.61 \pm 0.05$ & $0.68 \pm 0.08$ \\
Latitude $\left({ }^{\circ}\right)$ & $67.9 \pm 0.4$ & $31.8 \pm 2.2$ & $68.7 \pm 0.5$ & $-4.1 \pm 1.6$ & $68.9 \pm 0.7$ & $4.2 \pm 2.9$ \\
Longitude $\left({ }^{\circ}\right)$ & $345.0 \pm 0.8$ & $192.8 \pm 0.8$ & $349.1 \pm 1.5$ & $221.3 \pm 1.5$ & $330.5 \pm 2.4$ & $215.0 \pm 4.1$ \\
Period (fixed) & 11.55 & 11.39 & 11.55 & 11.39 & 11.55 & 11.39 \\
\hline$\chi^{2}$ (d.o.f.) & $39(167)$ & & $26(164)$ & & $92(166)$ & \\
& $1143(492)$ & & $4254(492)$ & & $1212(492)$ & \\
\hline
\end{tabular}

fixed in order to minimise the number of free parameters. The result (see Table 3) indicates changes in the starspot coverage that occur within less than a few rotation periods. The fit to the third dataset is of a lower quality than those to the first and second dataset since rapid changes in the lightcurve slope occur at the beginning of the last 12 day period that cannot be reproduced by the model. Also, the shape of the light curve during the second period is different from that of the first and third period dataset (see Fig. 1). There appear to be some structure going out of the second minimum and going into the third minimum that cannot be described with a 2-spot model. This structure, well above the noise level, supports evidence (Unruh et al. 1995) that, on fast rotating active dwarfs, starspot structure and position could change in a few rotation periods. Even small evolutionary effects in active regions could be a limitation in assessing the contribution of Sun-like faculae to the broadband flux modulation of active dwarfs.

Acknowledgements. I am grateful to the anonymous referee for the helpful comments that allowed me to improve the paper.

\section{References}

Aigrain, S., Favata, F., \& Gilmore, G. 2004, A\&A, 414, 1139

Baliunas, S. L., Vaughan, A. H., Hartmann, L., et al. 1983, ApJ, 275, 752

Baliunas, S. L., Donahue, R. A., Soon, W. H., et al. 1995, ApJ, 438, 269

Bean, J. L., Sneden, C., Hauschildt, P. H., et al. 2006, ApJ, 652, 1604

Benedict, G. F., McArthur, B. E., Gatewood, G., et al. 2006, AJ, 132, 2206

Biazzo, K., Frasca, A., Henry, G. W., et al. 2007, AJ, 656, 474

Chapman, G. A. 1987, J. Geophys. Res., 92, 809
Chapman, G. A., \& McGuire, T. E. 1977, ApJ, 217, 657

Chapman, G. A., Cookson, A. M., \& Dobias, J. J. 1994, ApJ, 432, 403

Chapman, G. A., Cookson, A. M., \& Dobias, J. J. 1997, ApJ, 482, 541

Claret, A., \& Gimenez, A. 1990, A\&A, 230, 412

Croll, B., Walker, G. A. H., Kuschnig, R., et al. 2006, ApJ, 648, 607

Eaton, J. A. 1992, LNP, 397, 15

ESA 1997, The Hipparcos Catalogue, ESA SP-1200

Fisher, G. F., Hall, D. S., Henry, G. W., et al. 1983, IBVS No. 2259

Fischer, G. F., \& Valenti, J. 2005, ApJ, 622, 1102

Foing, B., Crivellari, L., Vladilo, G., et al. 1989, A\&AS, 80, 189

Foukal, P. 1992, in The Solar Cycle, ed. K. Harvey (San Francisco: ASP), ASP Conf. Ser. 27, 439

Foukal, P., \& Lean, J. 1986, ApJ, 302, 826

Frey, G. J., Grim, B., Hall, D. S., et al. 1991, AJ, 102, 1813

Gaidos, E.J., Henry, G. W., \& Henry, S. M. 2000, AJ, 120, 1006

Gray, D. F., \& Baliunas, S. L. 1995, ApJ, 441, 436

Greaves, J. S., Holland, W. S., Wyatt, M. C., et al. 2005, ApJ, 619, 187

Güdel, M., Guinan, E. F., \& Skinner, S. L. 1997, ApJ, 483, 947

Hatzes, A. P., Cochran, W. D., McArthur, B., et al. 2000, ApJ, 544, 145

Landi, E., Landini, M., \& Del Zanna, G. 1997, A\&A, 324, 1027

Lanza, A. F., Rodono, M., \& Pagano, I. 2004, A\&A, 425, 707

Lanza, A. F., Messina, S., Pagano, I., et al. 2006, AN, 327, 28

Lockwood, G. W., Skiff, B. A., Henry, G. W., et al. 2007, ApJS, 171, 260

Marsden, S. C., Waite, I. A., Carter, B. D., et al. 2005, MNRAS, 359, 711

Messina, S., \& Guinan, E. F. 2002, A\&A, 393, 225

Moutou, C., Pont, F., Barge, P., et al. 2005, A\&A, 437, 335

Radick, R. R., Lockwood, G. W., Skiff, B. A., et al. 1998, ApJS, 118, 239

Ribarik, G., Olah, K., \& Strassmeier, K. G. 2003, AN, 324, 202

Rucinski, S. M., Walker, G. A. H., Matthews, J. M., et al. 2004, PASP, 116, 1093

Soderblom, D. R., \& Däppen, W. 1989, ApJ, 342, 945

Strassmeier, K. G., Pichler, T., Weber, M., et al. 2003, A\&A, 411, 595

Unruh, Y. C., Collier Cameron, A., \& Cutispoto, G. 1995, MNRAS, 277, 1145

Vaughan, A. H., Baliunas, S. L., Middlekoop, F., et al. 1981, ApJ, 250, 276

Walker, G. A. H., Matthews, J., Kuschnig, R., et al. 2003, PASP, 115, 1023

Walker, G. A. H., Croll, B., Kuschnig, R., et al. 2007, ApJ, 659, 1611

Weber, M, \& Strassmeier, K, G. 1998, A\&A, 330, 1029 